\title{
Enhanced Whale Optimization Algorithm for the Eye Movement Recognition
}

\author{
Vilas S.Gaikwad \\ Assistant Professor \\ Department of Computer Engineering JSPMNTC, Pune, Maharashtra, India
}

Vilasgaikwad11@gmail.com

\begin{abstract}
In recent days, the Electrooculography (EOG) signals triggers the Human Computer Interface (HCI) control system. On the basis of the EOG signal pattern, the recognition of eye movements is performed to preside over the HCI system and perform a definite job on the category of eye movement. By means of several associated assessments, this work proposes a new technique for the recognition of eye movement on the basis of EOG signal by exploiting the Enhanced Whale Optimization Algorithm (E-WOA) with the aid of Neural Network (NN). To minimize the error function from the classifier, the E-WOA model is exploited. The developed model performance is examined by evaluating the adopted technique with the traditional techniques. From the outcomes, it is exhibited that higher performance of developed technique with the minimization of error analysis and evaluation regarding the recognition in terms of various performance metrics such as Accuracy, Precision, FPR, Specificity,Sensitivity, FNR, NPV, F 1 Score and FDR.
\end{abstract}

Keywords:Classifier,Eye-movement;EOG, ECG, HCI, Neural network.

\begin{tabular}{ll}
\hline Nomenclature & \\
\hline Abbreviations & Descriptions \\
\hline BCI & Brain-computer interface \\
MRA & Multi-Resolution Analysis \\
NPV & Negative Predictive Value \\
KKLT & Kosambi-Karhunen-Loeve transformation \\
ERS & Event-Related Synchronization \\
EEG & Electroencephalogram \\
LM & Levenberg - Marquardt \\
SSVEP & Steady-State Visual Evoked Potentials \\
REM & Rapid eye movement \\
\hline
\end{tabular}

\section{Introduction}

The main target of the HCI intelligence is to enhance the user's purpose discernment which is derived from the HCI system. During the operation, it presents the theoretical basis and technical maintenance for the design of the adaptive HCI system and minimizing personal errors. In progress, widespread HCI purpose recognition techniques mostly rely on EEG signals or eye movement [1].

Eye-tracking systems tolerate massive possible of tracking eye movements throughout emotion reading, monitoring, the advertising perception, human activity recognition, visiting a website, HCI, cognition of sports, driving aid system, drivers' exhaustion exposure systems, so on. To implement an eye mouse, an eye-tracking system is exploited as well as it exploits as control signals to set up users to communicate with the interfaces of the system directly without the requirement for input devices such as keyboard or mouse for effortlessness of exploit of a computer for the disabled [2].

BCI is an application form of EEG signals that can set up a communication association amid the external devices as well as the human brain, hence allows the brain to control external devices. In fields like medicine neurobiology, psychology the BCI is used.

MI EEG shows off the kind of noninvasiveness, flexibility, minimum environmental needs, and maximum resolution. Hence, MI is considered as the extensive application form of BCI. During motion 
imagination, the EEG signal spectrum power will differ with the MI task content that is known as the ERS/ERD. The EEG signal is used to extract the features and exploit the ML approach for classification purposes. For motor, imaging recognition performs the average kappa value. In previous studies, the EEG signals are collected to carry out the data classification as well as feature extraction to comprehend the UAV remote control via BCI. Nevertheless, these purpose recognition techniques merely rely on EEG signals without the benefits of combining eye movement signals of that identification precise rate requires enhancement further [3].

Apart from this, EOG on the basis of HCI models can similarly be used to control several gadgets like console, TV, wheelchair, etc which presents a higher probability for all disabled individuals continuing with Locked-in Syndrome and Paralysis ALS as well as other dynamic ailments, who possess solemn problems in the motor system so far are fit for creating movements of eye as it were. Such models present improved communication and control more than the environs, minimizes the dependence and creates life additional pleasant for the patients aforesaid, then improving in the general benchmark of their life.

The main objective of this paper is to work on the recognition of different human eye movements. Moreover, the meta-heuristic optimization model is proposed named enhanced WOA method to minimize the error function as well as for the classification, the NN classifier is exploited.

\section{Literature Review}

In 2020, Yahui Wang et al [1], investigated the association amid the model decision making as well as the movements of the eye and response of EEG. To classify the diverse EEG features as well as to evaluate four diverse techniques were used. Moreover, in order to make the decision outcomes, two techniques were exploited for the selection of EEG features. Here, a multimodal integration scheme was exploited in order to model the recognition of decision-making. Moreover, EEG response data, as well as eye tracking, was exploited for the input dataset.

In 2017, Minrui Zhao et al [2], developed a new technique for purpose recognition, on the basis of eye movement and EEG in HCI. The integration of eye movement, as well as EEG, permits the maximum of the benefits of the two physiological signals. EEG signals and movement of an eye were gathered for the extraction of feature network of recognition for ML pattern was considered as the input for the objective recognition. Using the decision level integration the ultimate recognition outcome was obtained.

In 2019, Christoph Huber-Huber et al [3], worked on the saccadic eye movement. On the basis of the tangential glance of the saccadic target, here the main probable description for this occurrence was that the visual system forecasts imminent input athwart saccadic eye movements. By exploiting concurrent EEG and eye-tracking the experiment was examined. To inverted face stimulus or peripheral upright, participants performed cued saccades.

In 2017, Jing Chen et al [4], worked on the notice throughout smooth recreation was centered right on the allocated preferentially or pursuit target previous to it. By exploiting a secondary task, Attention deployment was formerly probed, which may have changed concentration allotment and lead to conflicting judgments. In the nonattendance of any secondary probing task to measure concentration allocation, the frequency-tagged SSVEP was measured. At a variety of positions in advance or following the tracking down the target, the performance was computed.

In 2017, Nan-Hung Lin et al [5], introduced an intelligent REM sleep recognition model which needs merely a single EEG channel. The developed model differentiates itself from preceding mechanical sleep performance techniques by developing two auxiliary feature sets to aid solve problems that occurred by interpersonal EEG signal diverse.

\section{EOG Signals: Optimized Feature Extraction}

\subsection{Signal Decomposition}

\section{i) Decomposition of EMCD}

Consider $g(r) ; r=1, \ldots \ldots ., R$ as the EOG signal by means of $R$ samples. $\left\{\left(q, g[q], q=1, \ldots \ldots, R_{q}\right\}\right.$ indicates the maxima sequence of $g(r)$, wherein, a number of maxima are indicated as $R_{q}$ and time indices of the maxima are indicated as $q$. Then, $\left\{(p, g[p]), p=1, \ldots ., R_{p}\right\}$ denotes the minimum series of $g(r)$, wherein, the time indices of minimum indicated as $p$ and the number of minimum denoted as $R_{p}$. From the minimum as well as maximum series, the superior, as well as inferior envelops, are substantiated. 
a) Superior Envelope: For the EOG signal, the superior envelope $g^{\sup }[r]$ represents the upper trend curve which passes via the maximum. Eq. (1) represents to interpolation the maximum; therefore the Bspline interpolation is exploited.

$$
\mathrm{g} \sup [\mathrm{r}]=\mathrm{B}_{\mathrm{s}}\{(\mathrm{q}, \mathrm{g}[\mathrm{q}]), \mathrm{r}\}, \mathrm{r}=\mathbf{1}, \ldots . \mathrm{R}
$$

b) Inferior Envelope: Eq. (2) defines the B-spline interpolation of the inferior envelope. For the EOG signal, the inferior envelope $g^{\inf }[r]$ the lower trend curve that transmits through minimum is considered 1 .

$$
g^{\inf }[r]=B_{s}\{(p, g[p]), r\}, r=1, \ldots ., R
$$

c) Mean curve: Eq. (3) defines Mean Curve ${ }^{\text {mean }}[\mathrm{r}]$ is referred as the average of both superior as well as inferior envelopes.

$$
\mathrm{g}^{\text {mean }}[\mathrm{r}]=(\mathrm{g} \sup [\mathrm{r}]+\mathrm{g} \inf [\mathrm{r}]) / \mathbf{2}, \mathrm{r}=\mathbf{1}, \ldots \ldots, \mathrm{R}
$$

d) Mode: Eq. (4) states mode $M_{o}$ which represents the average count of both maximum $R q$ and minimum $R_{p}$.

$$
\mathrm{M}_{\mathrm{O}}(\mathrm{g}[\mathrm{r}])=\left(\mathrm{R}_{\mathrm{q}}+\mathrm{R}_{\mathrm{p}}\right) / \mathbf{2}
$$

(e) EWF: Eq. (5) represents a simplification of mean curve determination proposes a novel EOG signal technique using extrema. Generally, EWF indicates the classification of discontinuous maxima and minima.

$$
\operatorname{EWF}(g[r])=\{(q, g[q]),(p, g[p])\}
$$

Generally, to indicate the mean curve, the EWF is exploited. Under the EOG principle, one whole sine wave presents only one mode that may be maximal else minimal therefore in conventional Fourier analysis the EWF model is ascertained. In eq. (6) as well as (7), the frequency $f^{\prime}$, as well as the empirical period, $T^{\prime}$ is stated.

$$
\begin{aligned}
& \mathrm{T}^{\prime}=\mathrm{R} / \mathrm{M}_{\mathrm{o}}(\mathrm{g}[\mathrm{r}]) \\
& \mathrm{f}^{\prime}=\mathrm{M}_{\mathrm{O}}(\mathrm{g}[\mathrm{r}]) \mathrm{R}
\end{aligned}
$$

On behalf of examining the model parameter, the EOG signal frequency, as well as empirical period estimation is effectual. In terms of this alleviation, the signal capability is improved and which are designed based on the oscillation. Generally, the EOG signal decomposition at several scale levels is the main contribution of decomposition of EMCD that gist the elucidated EWF. In addition, the EMCD basic model is the EWFs as well as they do not need any signal model knowledge. In the iterative procedure, the EMCD works and outcomes by means of the mean curve as the acquiesce

\section{ii)Dimensionality Reduction}

The mission of minimization of dimensionality frequently occurs in the recognition of statistical pattern as well as visual learning domains which intrinsically work on input data, high dimensional. The feature vector maximized size is fed to the implications of the harsh system which leads to exponential enlargement of classification storage space as well as a learning approach and running time. A linear mapping is performed by the PCA to minimize the original space by means of a number of point's dimensions. It subjected minimum loss of data; nevertheless, it optimistically keeps hold of the very significant variance. For dimensionality reduction, the EMCD decomposition process output $g\left[r^{\prime}\right]$ is subsequently subjected. Moreover, to minimize the dimension a process called PCA is exploited.

From $g\left[r^{\prime}\right]$, a group of $m$ data vectors $v_{1}^{\prime}, \ldots \ldots, v_{m}^{\prime}$ are selected and $v_{i}^{\prime}$ indicates the single troop scrutiny of variables $y$. Subsequently, to this, with all columns $l=1, \ldots . . y$, the empirical mean is computed as well as result mean value is exploited and the eq. (8) states the empirical mean vector $v$ with dimensions $y \times 1$, as well as eq. (9) states the mean deviation.

$$
\begin{aligned}
& \mathrm{v}[\mathrm{l}]=\frac{\mathbf{1}}{\mathrm{m}} \sum_{\mathrm{i}=\mathbf{1}}^{\mathrm{m}} \mathrm{Y}^{\prime}[\mathrm{i}, 1] \\
& \mathrm{B}=\mathrm{Y}^{\prime}-\mathrm{jv} \mathrm{v}^{\mathrm{T}}
\end{aligned}
$$

Wherein $B$ indicates $\mathrm{m} \times \mathrm{y}$ matrix and $\mathrm{j}$ indicates column vector $m \times 1$ of all $1 \mathrm{~s}: \mathrm{j}[\mathrm{i}]=\mathbf{1}, \mathrm{i}=\mathbf{1}, \ldots . \mathrm{m}$. Eq. (10) states the computation of covariance matrix $M^{c}$.

$$
\mathrm{M}^{\mathrm{c}}=\frac{1}{\mathrm{~m}-1} \mathrm{~B}^{*} \cdot \mathrm{B}
$$


Eq. (11) states the eigenvalues, and eigenvectors,are computed by valuing $X$ matrix that diagonalizes the $M^{c}$ covariance matrix.

$$
\mathrm{X}^{-1} \mathrm{M}^{\mathrm{c}} \mathrm{X}=\mathrm{A}
$$

wherein $A$ indicates the eigenvalues diagonal matrix of $M^{c}$. By sorting the eigenvector matrix column $X$ the eigenvalue matrix minimization of $A$ is attained. Eq. (12) states the cumulative energy content $c$ formulation. It summarizes the summation of the eigenvaluesenergy content from 1 via $l$.

$$
c[1]=\sum_{m=1}^{1} A[m, m], \text { for } 1=\mathbf{1}, \ldots . . . y
$$

By storing the column $C^{\prime}$ of $X$ as the D matrix nominates the eigenvectors subset. To choose the $C^{\prime}$ cost, the vector $c$ is exploited. $\hat{T}$ matrix column $\hat{T}=Z^{\prime} \cdot \mathrm{D}=\mathrm{KLT}\left\{\mathrm{Y}^{\prime}\right\}$ indicates vector and indicates the KKLT in $Y^{\prime}$ row matrix.

On the basis of eq. (13), $Z^{\prime}$ matrix is explained wherein $t=\{t[1]\}=\left\{\sqrt{\mathrm{M}^{\mathrm{c}}|1,1|}\right\}$ for $1=1, \ldots . . \mathrm{y}$ that is the ensuing dimensionally minimized signal $\mathrm{y}_{\mathrm{i}}[\mathrm{r}]$

$$
Z^{\prime}=\frac{B}{j \cdot t^{\hat{T}}}
$$

iii) Multi-wavelet decomposition: Finally, for several applications of image processing like wavelet packets as well as M-band filter banks, the wavelets are assigned which are considered as the important tools that are used for the oversimplification of MRA as well as a wavelet. The GHM Multi-wavelet system [6] is exploited with two pairs of wavelet functions as well as scaling to present the higher performance possibility. As eq. (14), let $\Phi(f)$ represents the scaling function that produces the multiresolution analysis. Eq. (15) indicates the matrix wavelet formulation is ascertained using the $r$ wavelets $\mathrm{w}_{\mathbf{0}}(\mathrm{f}), \ldots \ldots \ldots \ldots \ldots, \mathrm{w}_{\mathrm{r}-\mathbf{1}}(\mathrm{f})$, wherein $D_{k}$ and $C_{k}$ indicates the discrete wavelets and coefficients that related with the scaling functions.

$$
\begin{aligned}
& \Phi(f)=\sum_{k} C_{k} \Phi(2 f-k) \\
& w(f)=\sum_{k} D_{k} \Phi(2 f-k)
\end{aligned}
$$

\subsection{Feature Plane Optimization}

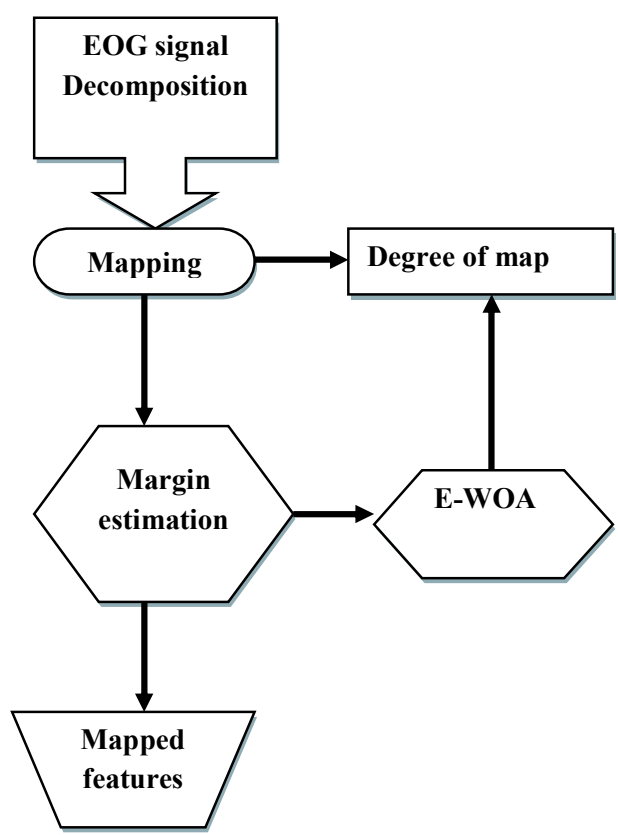

Fig. 1 Schematic model of Feature Mapping

Fig 1 exhibits the process of feature mapping. The decomposition method output $y_{i}[r]$ is subjected to a mapping plane block. As a result, through the multiplication of a weight function, it maps. Therefore, mapping procedures transform the signal to other domains as well as improve the margin. K-means 
clustering is exploited to calculate the mapped signalmargin. Chiefly, the clustered signal includes both accurate and erroneous clustered signals. The margin assessment is stated in eq. (16) that ascertains the mean of the accurately clustered signal, wherein, $C_{i}$ signifies the number of accurately classified signals as well as $i$ signifies the number of clusters.

$$
\mathrm{F}=\sum_{\mathrm{i}=\mathbf{1}}^{\mathrm{M}} \mathrm{C}_{\mathrm{i}}
$$

Furthermore, the optimization approach named, E-WOA is exploitedto precisely tune the margin, as well as the procedure bearing till conquering the targeted mapped feature.

\section{Enhanced WOA method for Eye Movements Recognition}

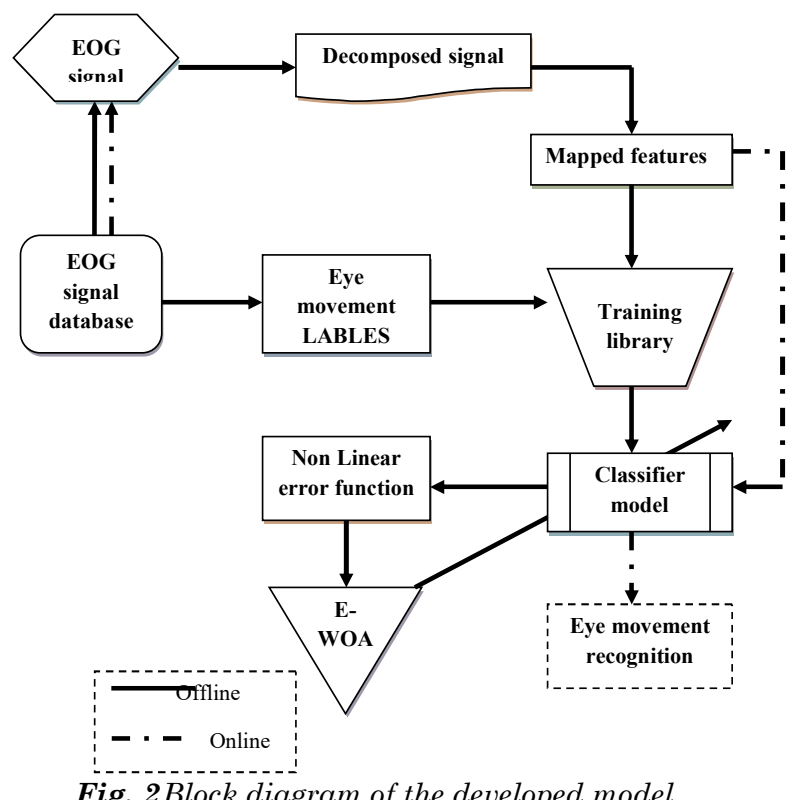

The schematic model of the developed method is shown in fig 2. From the EOG signal database, the EOG signal $g(r)$ is used. By exploiting the EMCD decomposition technique, $g(r)$ is decomposed and outcomes in the decomposed signal $g\left[r^{\prime}\right]$. For the minimization of the dimensions $g\left[r^{\prime}\right]$, the PCA is exploited. Subsequently, from the dimension minimization $y_{i}[r]$, the multi-wavelet features are extracted and used for the feature mapping.

In the training library, the mapped features are positioned. In the meantime, the eye movement labels that indicate the left movement, the right movement so on additionally the warehouse and retrieved in training library. As a result, in the NN classifier extracted features are used in the testing stage that is exploited to identify the precise eye movement. Finally, from the classifier, the nonlinear error function which happened is reduced by exploiting an advanced optimization approach named E-WOA.

\subsection{Classifier Model}

Using the NN classifier [8], the appropriate eye movement recognition can be clinched which is the ML approach. Moreover, to express the NN training LM approach is exploited [9]. The mathematical formulation of the NN is stated in Eq. (17), (18), (19), (20), and (21).

$$
\begin{aligned}
& y_{j}^{(h)}=N_{h}\left[u_{j}^{(h)} b_{h}+\sum_{i=1}^{Q_{I}^{(h)}} F_{i}^{(h)} v_{i j}(h)\right]: 0 \leq h \leq Q-1 \\
& \mathrm{w}=[\mathrm{u}, \mathrm{v}]
\end{aligned}
$$

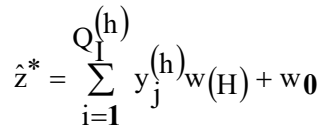




$$
\begin{aligned}
& \mathrm{w}^{*}=\underset{\mathrm{w}=[\mathrm{u} ; \mathrm{v}]}{\arg \min \left\{\log \left|\mathrm{z}^{*}-\hat{\mathrm{z}}^{*}\right|\right\}} \\
& |\mathrm{w}|=\sum_{\mathrm{h}=\mathbf{1}}^{\mathrm{Q}-\mathbf{1}}\left(\mathrm{Q}_{\mathrm{I}}^{(\mathrm{h}-\mathbf{1})} \times \mathrm{Q}_{\mathrm{I}}^{(\mathrm{h})}\right)+\mathrm{Q}_{\mathrm{I}}^{(\mathrm{h})}
\end{aligned}
$$

As aforesaid formulations, $w$ signifies the optimal probable weight coefficient of the network technique, $y_{j}^{(h)}$ signifies the output from the $j^{\text {th }}$ node of $h^{\text {th }}$ layer, $z^{*}$ signifies the actual output, $|w|$ indicates cardinality of $w, b_{h}$ signifies the bias, $Q_{I}^{(h)}$ signifies total number of output for the $h^{\text {th }}$ layer, and $N_{h}(\bullet)$ signifies the non-linear function of $h^{\text {th }}$ layer, $u$, and $v$ signifies the weight coefficients to strong the model with the suitable feature set, $w_{0}$ signifies bias weight as well as $w_{(H)}$ is the weight of the hidden neuron.

\subsection{Enhanced WOA}

The conventional WOA approach exploits fixed inertia weight therefore by means of maximizing the count of iterations; the conventional WOA does not alter the convergence speed along with the population position as well as the fixed inertia weight is not favorable to population leaping out of local best solution [7]. Hence, the dynamic inertia weight is developed in this work.

$$
\mathrm{w}(\mathrm{t})=\mathrm{w}_{\min } /\left(\mathrm{w}_{\max }-\mathrm{w}_{\min }\right) \times \mathrm{r} \times \mathrm{e}^{-\mathrm{t} / \mathrm{T}_{\max }}
$$

The inertia weight is developed into three location updating formulation of WOA

$$
\vec{X}(t+1)=\left\{\begin{array}{l}
w \times \vec{X} *(t)-\vec{A} \cdot \vec{D}_{1} \quad|\vec{A}|<\mathbf{1}, \text { rand }<0.5 \\
w \times \vec{X}_{\text {rand }} *(t)-\vec{A} \cdot \vec{D}_{2} \quad|\vec{A}| \geq \mathbf{1}, \text { rand }<\mathbf{0 . 5} \\
\vec{D}_{3}^{t} \cdot e^{b l} \cdot \cos 2 \pi+w \times \vec{X} *(t) \text { rand } \geq 0.5
\end{array}\right.
$$

$\vec{X} *(t)$ indicates the optimal location vector, $A^{\rightarrow}$ indicates the coefficient vectors, process. Even though the WOA possess better global convergence capability, it still has a few disadvantages in local search capability convergence speedand. Hence, this work adoptsRandomRyan Flight Search is used to update the ultimate location of whale aftereach iteration. On the basis of the Ryan distribution, levy flight is an arbitrary search. Currently, it is frequently exploited to enhance other optimization approaches. At last, the WOA approach 1

The position formulation of the WOA approach is developed by Levy Flight and it is altered to:

$$
\begin{aligned}
& \overrightarrow{\mathrm{X}}(\mathrm{t}+\mathbf{1})=\overrightarrow{\mathrm{X}}(\mathrm{t})+(\mathbf{2} \times \operatorname{rand}-\mathbf{1}) * \operatorname{levy}(\lambda) \\
& \text { levy } \sim \mathrm{u}=\mathrm{t}^{-\lambda} \mathbf{1}<\lambda \leq \mathbf{3}
\end{aligned}
$$

\section{Experimental Procedure}

In this section, the result analysis of the proposed and conventional models was demonstrated. Here, five kinds of eye movements have been included for the EOG signals, such as eye movement in, Left,Right, Down, Up, and Blinking was recorded. To model the EOG signal database three subjects were used. For eye movement, Manifold samples were used for each subject to make sure the data variety. For instance, 150 samples were exploited in this paper. Here, by exploiting the AD instruments, the EOG signals were recorded and by positioning $5 \mathrm{Ag} / \mathrm{AgCl}$ electrodes. To the normal EOG signal, the noise was augmented as well as the performance of the recognition was conducted for the several features by means of the classifier. Also, the analysis was carried out by exploiting various metrics.

The proposed method is compared with the conventional models such as Evolutionary approach (EA), Firefly (FF), Particle Swarm Optimization (PSO), Levenberg-Marquardt (LM), and Grey wolf Optimization (GWO) algorithms.

Fig 3 illustrates the graphical representation of the proposed and conventional models. Moreover, it describes the analysis by varying the sigma values. Moreover, Fig 3 illustrates the reducing performances that comprise FNR, FPR, and FDR of the developed model in identifying the eye movements like left movement, down movement, right, up, as we as the blinking of eyes. Here, the analysis shows that the identification of left movement of eyes with least FDR, FNR, and FPR. 


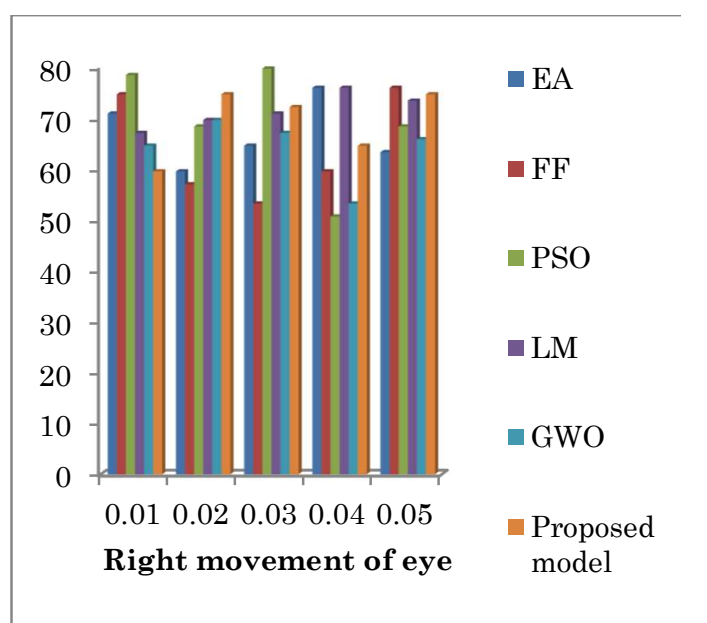

(a)

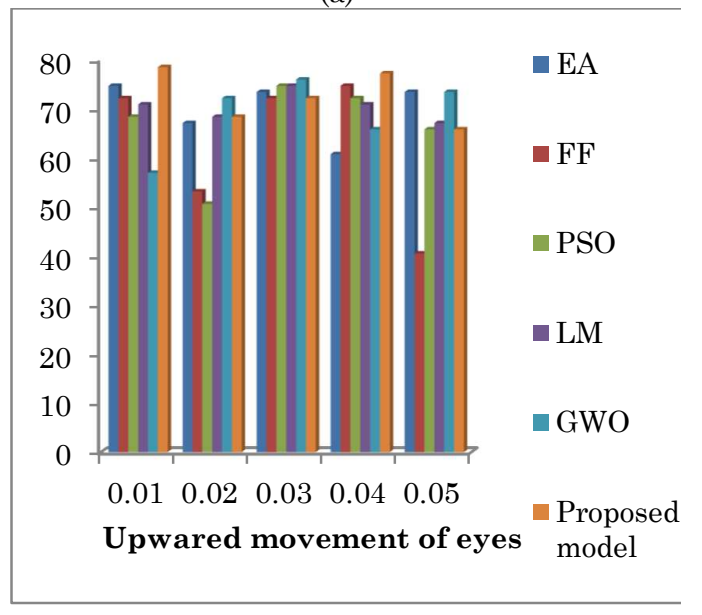

(c)

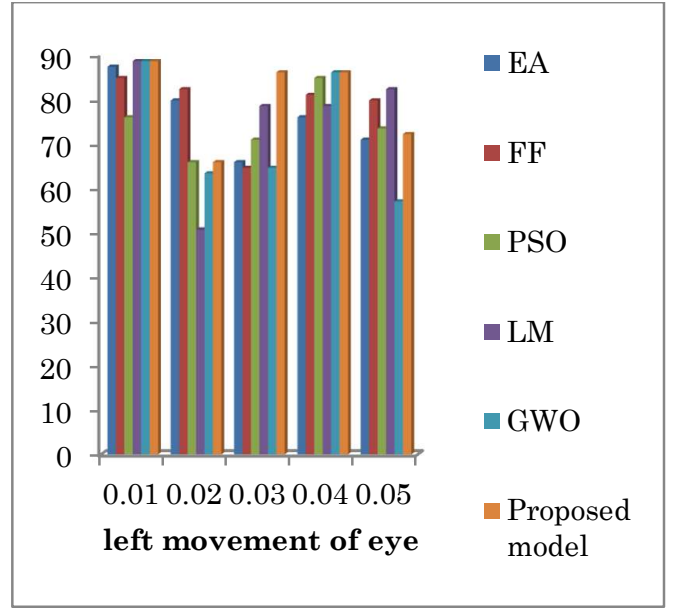

(b)

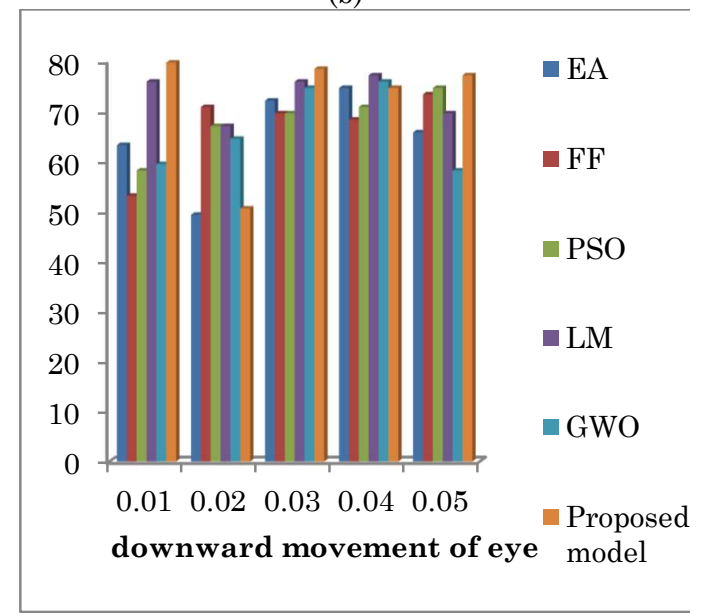

(d)

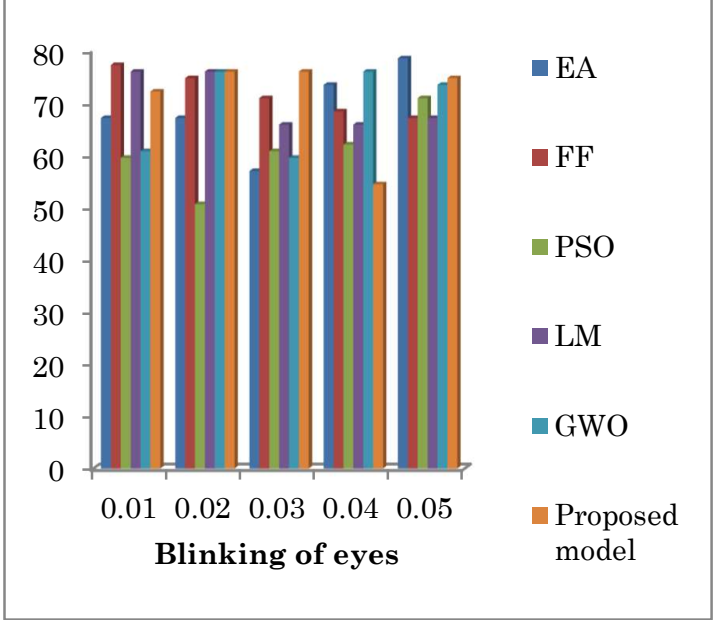

(e)

Fig. 3 Performance analysis of the proposed and conventional models (a) right movement of eyes (b) left movement of eyes(c) upward movement of eyes $(d)$ downward movement of eyes (e) Blinking of eye

\section{Conclusion}

Recently, to control the HCI system, the EOG signal is exploited which is diffuse as the developed model. Moreover, using several kinds of eye movements, the HCI system was controlled. A new optimization technique was proposed for the eye movement's recognition on the basis of the EOG signal patterns by exploiting E-WOA along with the NN approach. Using the proposed enhanced WOA approach, and also from the classifier, the error function was reduced. Subsequent to the experimentation, the developed 
model performance was evaluated with the conventional techniques. Finally, the performance was examined, the developed model posses higher performance through various metrics such as Accuracy, Precision, Specificity,Sensitivity, FNR, FPR, NPV, F 1 Score, and FDR. The overall analysis from the results exhibits that the adopted model performance states the betterment of the proposed model than the traditional techniques.

\section{Compliance with Ethical Standards}

Conflicts of interest: Authors declared that they have no conflict of interest.

Human participants: The conducted research follows the ethical standards and the authors ensured that they have not conducted any studies with human participants or animals.

\section{References}

[1] Yahui WangSuihuai YuJibo He,"Prediction of product design decision Making: An investigation of eye movements and EEG features", Advanced Engineering Informatics, vol.45, 10 April 2020.

[2] R. Benjamin Knapp, Jonghwa Kim,Elisabeth Andre"Physiological Signals and Their Use in Augmenting Emotion Recognition for Human-Machine Interaction",Emotion-Oriented Systems, vol. 172, pp.133-159.

[3] Christoph Huber-HuberAntimo BuonocoreDavid Melcher," The peripheral preview effect with faces: Combined EEG and eye-tracking suggests multiple stages of trans-saccadic predictive and non-predictive processing",vol. 200, pp. 344-362, NeuroImage28 June 2019.

[4] Jing ChenMatteo ValsecchiKarl R. Gegenfurtner,"Attention is allocated closely ahead of the target during smooth pursuit eye movements: Evidence from EEG frequency tagging", Neuropsychologia, vol. 102, pp. 206-216, 22 June 2017.

[5] Nan-Hung LinChung-Yao HsuChen-Wen Yen,"Detecting rapid eye movement sleep using a single EEG signal channel", Expert Systems with Applications, vol. 87, pp. 220-227, 13 June 2017.

[6] OzgurCelika, AhmetTekeb, H. BasakYildirima," The optimized artificial neural network model with LevenbergMarquardt algorithm for global solar radiation estimation in Eastern Mediterranean Region of Turkey, "Journal of Cleaner Production,vol.116,pp.1-12,10 March 2016.

[7] S. H. LiX. H. LuoL. Z. Wu,"An improved whale optimization algorithm for locating critical slip surface of slopes", Advances in Engineering Software3 May 2021.

[8] Yuying Chen,"Crowd Behaviour Recognition using Enhanced Butterfly Optimization Algorithm based Recurrent Neural Network", Multimedia Research, vol. 3, no. 3, July 2020.

[9] Raviraj Vishwambhar Darekar,Ashwinikumar Panjabrao Dhande," Emotion Recognition from Speech Signals Using DCNN with Hybrid GA-GWO Algorithm", Multimedia Research, vol 2, no 4, October 2019. 